\title{
Genital histomorphometrical evaluation and survey on reproductive traits of male camel (Camelus dromedarius) in relation to the pubertal age under extreme arid conditions
}

\author{
Djalel Eddine Gherissi ${ }^{1}{ }^{2}$, Meriem Boukhili ${ }^{1}$, Amina Gherissi ${ }^{3}$ \\ ${ }^{1}$ Institute of agronomic and veterinary sciences, University of Souk-Ahras, Algeria \\ ${ }^{2}$ Laboratory of Animal Productions, Biotechnologies and Health. University of Souk-Ahras, Algeria \\ ${ }^{3}$ Faculty of Natural Sciences and Life, University of El Tarf, Algeria
}

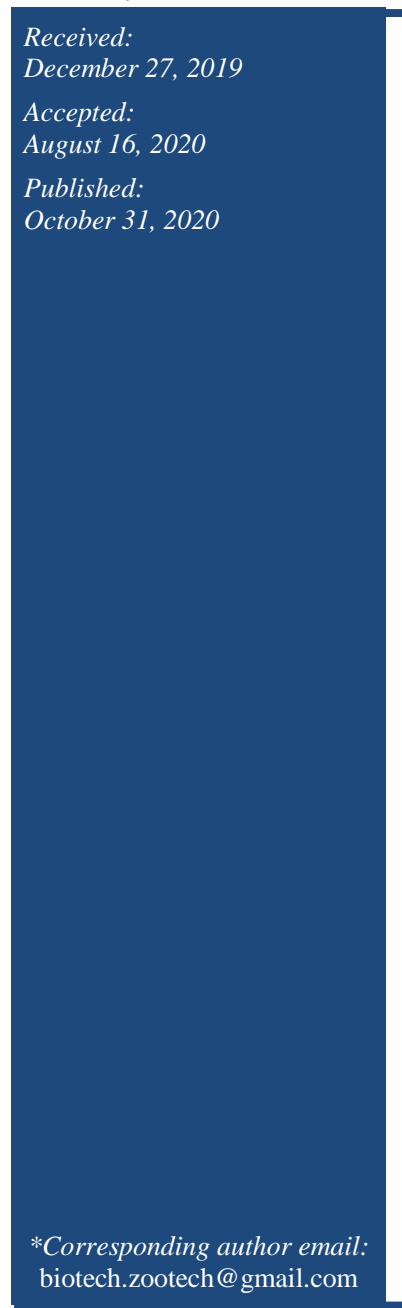

\begin{abstract}
The present study consists on age-related analysis of reproductive activity in male dromedary camel from extreme arid area. Five reproductive traits of 20 bull camels were recorded and computer-assisted histomorphometric evaluation of 48 paired testes were performed to establish quantitative developmental pattern in relation to their respective age. The pubertal changes were displayed mainly at 4 to 5 years old with increased sperm reserves, seminiferous tubules volume, diameter and total length, high relative volume of interstitial tissue components, high individual Leydig cells volume and total number Leydig cells. At this age the first reductional miotic splits and first spermatids and spermatozoa in the seminiferous lumen tubules were significantly developed leading to increase the mean germ cells number and tubular fertility indexes. Animals in this age category showed the highest proportion of first mating (50\%), while mating frequency and time were relatively higher. These reproductive traits were improved significantly with spermatogenesis efficiency in mature animals aged $>6$ years old. The testicular spontaneous degeneration was observed in animals ages $\geq 13$ years old. Our data showed long postnatal duration to onset spermatogenesis and endocrine compartment proliferation, that lead to delayed puberty (4 to 5 years), short duration of maximum activity ( 8 to10 years) and early genital degenerative involution ( from 13 years).
\end{abstract}

Keywords: Age, Dromedary camel, Leydig cells, Reproductive traits, Sertoli cells, Spermatogenesis

\section{How to cite this:}

Gherissi DE Boukhili M and Gherissi A, 2020. Genital histomorphometrical evaluation and survey on reproductive traits of male camel (Camelus dromedarius) in relation to the pubertal age under extreme arid conditions. Asian J. Agric. Biol. 8(4): 436-446. DOI: https://doi.org/10.35495/ajab.2019.12.591

This is an Open Access article distributed under the terms of the Creative Commons Attribution 3.0 License. (https://creativecommons.org/licenses/by/3.0), which permits unrestricted use, distribution, and reproduction in any medium, provided the original work is properly cited. 


\section{Introduction}

The dromedary camel population in Algeria had been multiplied by 2.06 between 1961 and 2018, reaching 381.882 heads (FAOSTAT, 2018). The evolutionary pattern of the world camel population varies across the countries; from its alarming decline in India to its astonishing growth in the Horn of Africa and Sahelian countries (Faye, 2016). The decline in camel growth rate is due to a reduction in arid land devoted to camel rearing, low herd fertility and a lack of selection programs (Faye, 2016).

Camel species is considered to have poor reproductive efficiency under natural conditions (Gherissi, 2015; Gherissi et al., 2020). Male puberty is defined as the moment of sexual behavioral activity associated with first ejaculation with enough sperm cells to accomplish fertilization (Tibary and Anouassi, 1997). The pubertal age in male dromedary camels is almost twice the age of other species of the same size e.g. the horse (Dhakal et al., 2012). In addition, pronounced heterogeneity of male camel sexual activity beginning and achievement was reported by some authors. The first mating age was 3 to 4 years in Egypt (El-Harairy and Attia, 2010) and 4 to 5 years in Iraq and Ethiopia (Hafez and Hafez 2001; Abd and Ibrahim, 2014; Mirkena et al., 2018). It can be reported by herder's voluntary delay until 6 years (Gherissi, 2015; Gherissi et al., 2020) and 8 years (Al Qarawi et al., 2001). The sexual abilities of the camel bull can increase up to 10 years, and then remain at a fairly high level more or less constant until the age of 14 to 20 years (Al Qarawi et al., 2001; Gherissi et al., 2020). The animal's genetic, growth, geographic area and study methods would the origin of these wide differences. During different pubertal stages, genital organs express a tissue and cellular remodeling which characterize the histo-functional evolution. These changes are determinant of the breeding ability and fertility, therefore it could be important to characterize normal testicular evolutionary aspects in offspring and to evaluate degenerating aspects of such important organs.

The aim of the present study is to provide a histofunctional analysis of camel testes and epididymis in order to bring critical discussion of the reproductive traits according to the different pubertal stages under extreme arid conditions. This would provide a basic data needed for a more rational management of camel bulls within camel herds.

\section{Material and Methods}

\section{Study area}

The present study was conducted over one year in $\mathrm{El}$ Oued area situated at the South-East of Algeria (lat.33$34^{\circ}$ Nandlon. $\left.6^{\circ}-8^{\circ} \mathrm{E}\right)$. This region is characterized by extreme arid climatic conditions (average altitude of $80 \mathrm{~m}$, average annual temperature of around $25^{\circ} \mathrm{C}$, max $52^{\circ} \mathrm{C}$, min $2{ }^{\circ} \mathrm{C}$, average annual precipitation of $80 \mathrm{~mm}$ : $\max 160 \mathrm{~mm}$, $\min 20 \mathrm{~mm}$ ).

\section{Male camel reproductive traits}

Data about reproductive activity of 20 bull camels belonging to 14 pastoral camel herds were collected through livestock visit investigation and participatory appraisal using "Progeny History Testing" (PHT) as described by Keskes et al. (2013) (supplementary figure 1). The strong reliance of the pastoral herders and their families with the camel herd and their dependency in different aspects of life and activities on camel species facilitated to remember the history and main events of each animal in an exact time-scheduled manner. The following parameters were recorded: Age to First Mating AFM (years); Number of Mating for Pregnancy per female (NMP); Mating Time (TM); Male to Female Ratio (RMF) and Culling Age (CA)

\section{Slaughtered animals and samples collection}

Paired testes and epididymis were collected from 48 slaughtered Sahraoui male camels aged from 2 to over 13 years old. No case slaughter for sub-fertility or infertility was mentioned. The studied animals were distributed over 8 age classes ( 2 years, 3 years, 4 years, 5 years, 7 years, 8 years, 10 years, $>13$ years) at the rate of 6 animals per class ( 3 animals in the breeding season and 3 animals in the non-breeding season). The animals were checked carefully before slaughter to guarantee their health and wellbeing statue. All disabled and diseased animals and those with low body conditions or abnormalities in the scrotal region were discarded form this study. The collected testes, with their epididymis were separated from surrounding tissues, measured and fixed in formaldehyde (10\%).

\section{Testicular measurements}

For each paired testes the averages of testicular length (ATL), testicular thickness (ATT) and testicular width (ATW) were measured in centimeters by using a vernier caliper (Gherissi et al., 2014). The paired testicular volume $\left(\mathrm{PTV} \mathrm{cm}^{3}\right)$ was calculated using the 
equation for ellipsoid volume: 4/3 $\pi$ TLxTWxTT, TL.TW.TT $=$ axes of ellipsoid (Al-Saiady et al., 2013).

\section{Quantitative histology}

All formalin fixed specimens were dehydrated, embedded in paraffin wax and sectioned on microtome at thickness $5 \mu \mathrm{m}$ then they were stained by haematoxylin and eosin (H\&E) stain according to descriptions of Gherissi et al. (2018b). Histological sections were examined using binocular microscope Optika B-600B equipped with a digital camera HiROCAM (5MP) and fitted with a micrometer eyepiece at 100, 400 and 1000 magnifications. AxovisonRel 4.6 (Carl Zeiss, Thornwood, NY) and Image J 1.45S (NIH, USA) processing software were used for quantitative histomorphometry of epididymis, testicular exocrine and endocrine compartments taking into account the analysis of volume (3D) by Weibel microscope reticle, surface (2D) by semisedimented segmentation, length (size 1) by micrometric measurement, and number $(0$ dimensions) by point counting (Supplementary Figures 2 to 5). The stereological techniques and the histomorphometric methods used for this study were performed according to the recommendations of Gherissi et al. (2016, 2018b). Therefore, the following parameters were calculated (supplementary tables 1 , 2): Epididymal filling level (ER\%), Thickness of epididymal pseudo-stratified columnar epithelium (TEE $\mu \mathrm{m}$ ), Epididymal stereocilia thickness (EST $\mu \mathrm{m})$, Testicular volume occupied by the interstitial tissue (VIT $\mathrm{cm}^{3}$ ), Testicular volume occupied by seminiferous tubules (VST $\mathrm{cm}^{3}$ ), Ratio seminiferous tubules volume to Interstitial tissue volume (VST/VIT), Volume percentage of the interstitial tissue occupied by Leydig cells, conjunctive tissue and blood vessels (VLC, VCT, VBV in $\mathrm{cm}^{3}$ and \%), Diameter of Leydig cell's nucleus $\left(\mathrm{D}_{\mathrm{NyLC}} \mu \mathrm{m}\right)$, Individual Leydig cell volume $\left(\mathrm{V}_{\mathrm{LC}} \mu \mathrm{m}^{3}\right)$, Relative area occupied by the seminiferous epithelium in the seminiferous tubules section (SE/ST \%), Relative area occupied by the lumen in the seminiferous tubule section (L/ST \%), Seminiferous tubule diameter (STD $\mu \mathrm{m}$ ), Seminiferous tubule lumen diameter (DLST $\mu \mathrm{m}$ ), Seminiferous tubule epithelium thickness (STET $\mu \mathrm{m}$ ), Total length of seminiferous tubules (TLST $\mu \mathrm{m}$ ), Enumeration of seminiferous epithelium cells (sg: spermatogonia, scyI: Spermatocyst I, scyII: Spermatocyst II, std: Spermatides, Sptz: Spermatozoa, srt: Sertoli cells), Tubular fertility indexes (TGC: total number of germ cells per seminiferous tubule section, Germ/Srt: Ratio of germ cells to Sertoli cells per seminiferous tubule section, $\mathrm{ST}_{\mathrm{Std}: \mathrm{Sptz}} \%$ : Percentage of ST containing spermatids and/or spermatozoa), Number of Leydig cells per testes $\left(\mathrm{N}_{\mathrm{LC} / \mathrm{T}} 10^{9}\right.$ cells), Crude number of Sertoli cells (Srt/T-CN $10^{10}$ cells) and True number of Sertoli cells (Srt/T-TN $10^{10}$ cells).

\section{Statistical analysis}

The data analysis was performed using the statistical analysis system (SPSS program, version 20.0, 2013). For each dependent variable, the normality was checked separately for parametric tests using Kolmogorov-Smirnov test. The one-way analysis of variance (ANOVA) was applied to find out the significant differences among different age groups. The Post-hoc Dancun test was used to compare means when moving from one age group to the next one. The effect of the age on the reproductive traits was analyzed using Khi-2 test. The statistical significance is calculated at $\mathrm{P}$ $<0.05, \mathrm{P}<0.01$ and $\mathrm{P}<0.001$.

\section{Results}

Reproductive traits in relation to male camel age The average age at first mating (AFM) and the culling age $(\mathrm{CA})$ are $3.55 \pm 1.19$ years and $15.30 \pm 2.47$ years, respectively (Table 1).

Table-1. Descriptive statistics of male camel's reproductive traits in South-East Algeria

\begin{tabular}{|l|c|c|c|c|c|c|c|c|}
\hline Variable & & & N & Mean & SD & Median & Min & Max \\
\hline Age at first mating & Years & AFM & 20 & 42.6 & 14.28 & 48 & 24 & 72 \\
\hline Mating/pregnancy & Integer & NMP & 20 & 2.09 & 1.03 & 2 & 1 & 5 \\
\hline Mating time & Minutes & TM & 20 & 17.50 & 6.48 & 15 & 10 & 30 \\
\hline Ratio male/female & & RMF & 20 & $1: 40$ & 24.01 & $1: 52$ & $1: 20$ & $1: 100$ \\
\hline Age at culling & Years & CA & $14^{*}$ & 15.30 & 2.473 & 15 & 12 & 20 \\
\hline
\end{tabular}

* Descriptive statistics for rerproductive tarits of 14 camel herds. 
Fifty percent $(50 \%)$ of male camels breed for the first time at the age of 4 to 5 years, $25 \%$ breed at 2 to 3 years old and $15 \%$ at 5 to 6 years old. Only $10 \%$ of male dromedaries are prone to first mating at an age $\geq$ 6 years (Figure 1). The culling age of the camel bulls as reported by the herders is $64.3 \%$ at an age $>15$ years, $14.3 \%$ for animals aged $<13$ years old and 21.42 $\%$ at the age of $13-15$ years old (Figure 1).
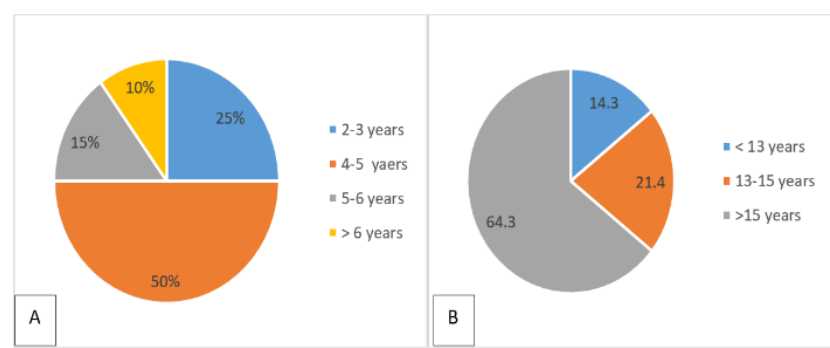

Figure-1. Frequency of bull camels $(n=20)$ according to their age at first mating (years) (A) and culling age (years) (B) in the South East Algeria

The average mating frequency (NMP) is $2.9 \pm 1.03$ and the average mating time (TM) is $17.50 \pm 6.48$ minutes (Table 1). The camel bull age shows significant effect on these reproductive traits $(\mathrm{P}<0.01)$. The NMP $\geq 3$ had been recorded in two age classes of 2-3 years and $3-4$ years (100\% and 20\%, respectively) (Figure 2A). All male camels aged $\geq 5$ years mate and fecund females pregnancy with 1 or 2 mating and mating time per female always < 15 minutes. However, 40 and $30 \%$ of camel bulls' aged 2 to 3 years and 4 to 5 years, respectively, mate with an average time $>20$ minutes (Figure 2B).

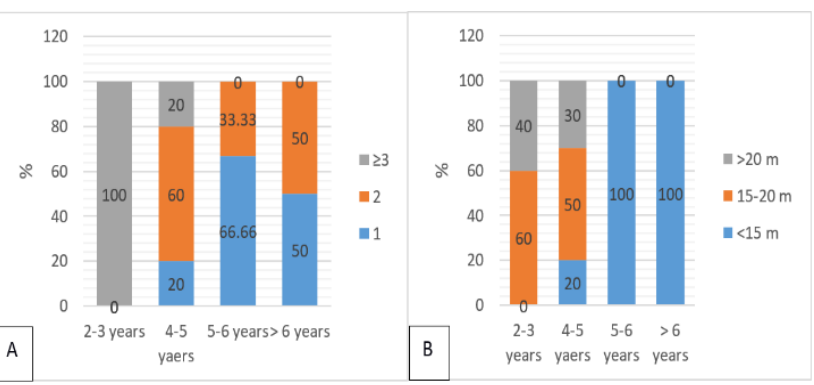

Figure-2. Number of mating for pregnancy per female (NMP) and mating time (MT) according to bull camel age $(n=20)$ in the South-East Algeria

Ratio male to female camels (RMF) during the breeding season is 1:40 \pm 24.01 (Table 1). This ratio is variable according to the age of the male dromedary $(\mathrm{P}<0.01)$. Camel bulls aged 2 to 3 years are used with
RMF <1:20 (40\%) and 1:20 to $1: 40 \quad(60 \%)$; respectively. However, $20 \%$ of camels aged 4 to 5 years old breed with ratio $\geq 1: 40$. This proportion reaches $33.33 \%$ and $100 \%$ for males aged 5 to 6 years and $>6$ years, respectively (Figure 3 ).

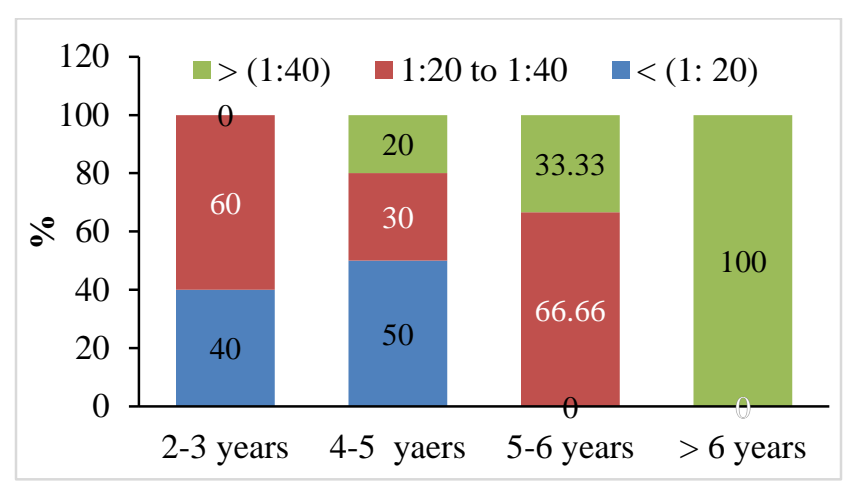

Figure-3. Male to female ratio (MFR) according to bull camel age $(n=20)$ in the South-East Algeria)

\section{Morpho-functional analysis \\ Testicular volume, seminiferous and interstitial compartments volume}

The PTV, VST and VIT display significant low scores in animals aged between 2 and 4 years and increase significantly at 5 years old then they reach maximal value at 10 years old.

Table-2. Paired testicular volume, seminiferous tubules and interstitial tissue volume and ratio of seminiferous to interstitial tissue volumes according to the age of Sahraoui male camel

\begin{tabular}{|c|c|c|c|c|}
\hline Age (years) & PTV $\left(\mathrm{cm}^{3}\right)$ & VIT $\left(\mathrm{cm}^{3}\right)$ & VST $\left(\mathrm{cm}^{3}\right)$ & VST/VIT \\
\hline $2(n=6)$ & $35.23 \pm 21.94^{\mathrm{a}}$ & $22.03 \pm 13.63^{\mathrm{a}}$ & $17.13 \pm 11.44^{\mathrm{a}}$ & $0.88 \pm 0.38^{\mathrm{a}}$ \\
\hline $3(n=6)$ & $25.61 \pm$ & $14.13 \pm 6.85^{\mathrm{a}}$ & $11.48 \pm 6.68^{\mathrm{a}}$ & $0.94 \pm 0.60^{\mathrm{a}}$ \\
\hline $4(n=6)$ & $25.16 \pm 11.65^{\mathrm{a}}$ & $11.88 \pm 7.31^{\mathrm{a}}$ & $19.56 \pm 12.63^{\mathrm{a}}$ & $1.64 \pm 0.40^{\mathrm{c}}$ \\
\hline $5(n=6)$ & $95.01 \pm 42.99^{c}$ & $52.24 \pm 19.55^{\mathrm{c}}$ & $45.76 \pm 25.22^{\mathrm{c}}$ & $0.95 \pm 0.44^{\mathrm{a}}$ \\
\hline $7(n=6)$ & $138.23 \pm 31.16^{\mathrm{d}}$ & $76.18 \pm 32.63^{\mathrm{d}}$ & $62.05 \pm 8.07^{\mathrm{d}}$ & $1.01 \pm 0.55^{\mathrm{c}}$ \\
\hline $8(n=6)$ & $170.63 \pm 65.17^{\mathrm{d}}$ & $83.93 \pm 38.53^{d}$ & $92.05 \pm 39.41^{\mathrm{e}}$ & $1.22 \pm 0.59^{c}$ \\
\hline $10(n=6)$ & $246.74 \pm 1.45^{\mathrm{e}}$ & $146.16 \pm 21.45^{\mathrm{e}}$ & $99.86 \pm 21.49^{\mathrm{e}}$ & $0.72 \pm 0.30^{\mathrm{a}}$ \\
\hline$\geq 13(n=6)$ & $133.50 \pm 65.17^{\mathrm{d}}$ & $72.46 \pm 56.55^{\mathrm{d}}$ & $61.04 \pm 13.16^{\mathrm{d}}$ & $1.36 \pm 0.96^{\mathrm{c}}$ \\
\hline
\end{tabular}

Testicular volume occupied by the interstitial tissue, $\operatorname{VST}\left(\mathrm{cm}^{3}\right)$ Testicular volume occupied by seminiferous tubules, VST/VIT: Ratio seminiferous tubules volume to interstitial tissue volume. Values with dissimilar letters within columns are significantly different $(c-d$, $d-e: P<0.05$; a-c, c-e: $P<0.01 ; a-d$, a-e: $P<0.001)$.

The VST/VIT ratio $(1.64 \pm 0.40)$ shows particular 
increase of VST at 4 years (Table 2). It is significantly decreased at 10 years old in relation to the relative VIT compared to VST (59\% and $41 \%$ of VT volume) reaching $0.72 \pm 0.30$. Finally, VIT and VST are significantly increased in male camels aged $\geq 13$ years old $(\mathrm{VST} / \mathrm{VIT}=1.36 \pm 0.96)$.

Table-3. Epididymal parameters changes according to the age of Sahraoui male camel

\begin{tabular}{|c|c|c|c|}
\hline $\begin{array}{c}\text { Age } \\
(\text { years })\end{array}$ & ER $(\boldsymbol{\%})$ & TEE $(\boldsymbol{\mu m})$ & EST $(\boldsymbol{\mu m})$ \\
\hline $\mathbf{2}(\mathbf{n = 6})$ & $20.64 \pm 19.13^{\mathrm{a}}$ & $19.19 \pm 10.37^{\mathrm{a}}$ & $11.90 \pm 6.69^{\mathrm{a}}$ \\
\hline $\mathbf{3}(\mathbf{n}=\mathbf{6})$ & $22.56 \pm 13.87^{\mathrm{a}}$ & $16.67 \pm 14.60^{\mathrm{a}}$ & $9.32 \pm 5.78^{\mathrm{a}}$ \\
\hline $\mathbf{4}(\mathbf{n = 6})$ & $38.73 \pm 26.36^{\mathrm{b}}$ & $22.36 \pm 14.05^{\mathrm{a}}$ & $14.04 \pm 7.86^{\mathrm{b}}$ \\
\hline $\mathbf{5}(\mathbf{n = 6})$ & $40.63 \pm 11.76^{\mathrm{b}}$ & $28.57 \pm 13.00^{\mathrm{a}}$ & $17.71 \pm 7.59^{\mathrm{b}}$ \\
\hline $\mathbf{7}(\mathbf{n}=\mathbf{6})$ & $37.80 \pm 10.89^{\mathrm{b}}$ & $39.50 \pm 8.92^{\mathrm{b}}$ & $14.68 \pm 4.47^{\mathrm{b}}$ \\
\hline $\mathbf{8}(\mathbf{n = 6})$ & $40.64 \pm 17.01^{\mathrm{b}}$ & $30.54 \pm 14.58^{\mathrm{b}}$ & $13.98 \pm 6.10^{\mathrm{b}}$ \\
\hline $\mathbf{1 0}(\mathbf{n = 6})$ & $61.62 \pm 3.94^{\mathrm{c}}$ & $41.33 \pm 9.70^{\mathrm{b}}$ & $17.67 \pm 3.05^{\mathrm{b}}$ \\
\hline$\geq \mathbf{1 3}(\mathbf{n}=\mathbf{6})$ & $19.65 \pm 5.70^{\mathrm{a}}$ & $29.34 \pm 9.70^{\mathrm{a}}$ & $17.64 \pm 4.50^{\mathrm{b}}$ \\
\hline
\end{tabular}

ER (\%): Epididymal filling level, TEE $(\mu \mathrm{m}):$ Thickness of epididymal pseudo-scarified columnar epithelium, EST $(\mu \mathrm{m})$ : Epididymal stereocilia thickness. Values with dissimilar letters within columns are significantly different ( $a-b, b-c: P<0.05 ; a-c: P<0.01)$.

\section{Epididymal histomorphometric parameters}

The age has a very highly significant effect on all epididymal parameters ( $\mathrm{P}<0.001$; Table 3 ). Low scores of TEE are recorded between 2 and 5 years old $(19.19 \pm 10.37-28.57 \pm 13.00 \mu \mathrm{m})$. The first significant increase of this parameter is observed at 7 years old $(39.50 \pm 8.92 \mu \mathrm{m}, \mathrm{P}<0.05)$. The EST and ER are low until 3 years old and then they increase significantly from the 4th year old $(14.04 \pm 7.86 \mu \mathrm{m}$, $38.73 \pm 26.36 \%$ respectively; $\mathrm{P}<0.05)$. The maximum values of TEE and EST and SR are recorded in camels aged 10 years old. Nevertheless, particular decreases of ER and EST are observed in animals aged $\geq 13$ years old $(\mathrm{P}<0.05)$.

\section{Endocrine compartment parameters}

Testes form animals aged 5 years old show first significant increase in VLC, VCT and VBV $(\mathrm{P}<0.05$; Table 4). They displayed a continual and gradual evolution of the mentioned parameters up to reach their maximal levels in older animals (Table 4).

However, the relative volume of interstitial tissue constituents did not vary significantly in relation to the animal's age. The prominent increase of $\mathrm{V}_{\mathrm{LC}}$ and $\mathrm{D}_{\mathrm{NyLC}}$ was observed at 4 years old $(\mathrm{P}<0.01)$. This parameters remain higher in animals aged $\geq 13$ years (Table 5).

\section{Exocrine compartment parameters}

The DLST increases at 3 years old $(67.26 \pm 44.01 \mu \mathrm{m}$; $\mathrm{P}<0.05)$, STD and STET increase significantly at 4 years old about $(139.59 \pm 34.35 \mu \mathrm{m}$ and $44.42 \pm$ $13.81 \mu \mathrm{m}$, respectively) and TLST display first significant increase at 5 years old $(2505.89 \pm 135.68$ $\mathrm{m}, \mathrm{P}<0.05)$.

STD, DLTS, STET reach maximal scores in males aged between 8 and 10 years old. The STD, STET have been deceased significantly in camel males aged $\geq 13$ years old but DLST and TLST remain thereafter at high levels (Table 6).

Spermatogenesis is limited to spermatocysts I stage in camels aged 1 to 3 years (supplementary figure 6). The first reductional miotic splits generating spermatocysts II among ST germ cells are recorded in males of 4 year old $(\mathrm{P}<0.05$; Table 7$)$.

Table-4. Volume and relative volume of interstitial tissue components according to the age of Sahraoui male camel

\begin{tabular}{|l|c|c|c|c|c|c|}
\hline Age (years) & VLC $\left(\mathbf{c m}^{\mathbf{3}}\right)$ & VCT $\left(\mathbf{c m}^{\mathbf{3}}\right)$ & VBV $\left(\mathbf{c m}^{\mathbf{3}}\right)$ & VLC/VIT (\%) & VCT/VIT $(\%)$ & VBV/VIT (\%) \\
\hline $\mathbf{2}(\mathbf{n = 6})$ & $10.07 \pm 7.72^{\mathrm{a}}$ & $1.68 \pm 1.40^{\mathrm{a}}$ & $0.86 \pm 0.53^{\mathrm{a}}$ & $79.54 \pm 8.35^{\mathrm{a}}$ & $12.46 \pm 5.30^{\mathrm{a}}$ & $7.48 \pm 3.99^{\mathrm{a}}$ \\
\hline $\mathbf{3 ( n = 6 )}$ & $9.13 \pm 7.06^{\mathrm{a}}$ & $1.90 \pm 1.35^{\mathrm{a}}$ & $0.90 \pm 0.67^{\mathrm{a}}$ & $76.03 \pm 6.81^{\mathrm{a}}$ & $16.32 \pm 6.25^{\mathrm{a}}$ & $7.64 \pm 2.30^{\mathrm{a}}$ \\
\hline $\mathbf{4}(\mathbf{n = 6})$ & $7.71 \pm 3.16^{\mathrm{a}}$ & $2.53 \pm 0.83^{\mathrm{a}}$ & $0.75 \pm 0.36^{\mathrm{a}}$ & $69.17 \pm 7.18^{\mathrm{a}}$ & $23.33 \pm 6.57^{\mathrm{a}}$ & $6.86 \pm 2.35^{\mathrm{a}}$ \\
\hline $\mathbf{5}(\mathbf{n = 6})$ & $39.24 \pm 17.81^{\mathrm{c}}$ & $8.18 \pm 5.79^{\mathrm{b}}$ & $3.40 \pm 2.33^{\mathrm{c}}$ & $77.60 \pm 9.20^{\mathrm{a}}$ & $15.40 \pm 8.67^{\mathrm{a}}$ & $7.00 \pm 3.63^{\mathrm{a}}$ \\
\hline $\mathbf{7 ( \mathbf { n } = \mathbf { 6 } )}$ & $48.76 \pm 28.41^{\mathrm{e}}$ & $10.67 \pm 2.14^{\mathrm{b}}$ & $3.59 \pm 1.78^{\mathrm{c}}$ & $74.05 \pm 8.80^{\mathrm{a}}$ & $20.16 \pm 8.55^{\mathrm{a}}$ & $5.80 \pm 2.20^{\mathrm{a}}$ \\
\hline $\mathbf{8 ( n = 6 )}$ & $70.50 \pm 33.45^{\mathrm{e}}$ & $8.81 \pm 3.23^{\mathrm{b}}$ & $5.40 \pm 5.00^{\mathrm{c}}$ & $89.05 \pm 4.09^{\mathrm{a}}$ & $4.97 \pm 3.41^{\mathrm{a}}$ & $5.49 \pm 2.94^{\mathrm{a}}$ \\
\hline $\mathbf{1 0}(\mathbf{n = 6})$ & $55.60 \pm 14.17^{\mathrm{e}}$ & $3.80 \pm 1.53^{\mathrm{a}}$ & $4.20 \pm 1.64^{\mathrm{c}}$ & $86.97 \pm 3.68^{\mathrm{a}}$ & $6.13 \pm 2.26^{\mathrm{a}}$ & $6.90 \pm 3.39^{\mathrm{a}}$ \\
\hline$\geq \mathbf{1 3}(\mathbf{n}=\mathbf{6})$ & $122.49 \pm 6.60^{\mathrm{a}}$ & $19.03 \pm 3.08^{\mathrm{d}}$ & $8.89 \pm 4.43^{\mathrm{e}}$ & $81.53 \pm 2.36^{\mathrm{a}}$ & $12.69 \pm 2.04^{\mathrm{a}}$ & $5.78 \pm 2.73^{\mathrm{a}}$ \\
\hline
\end{tabular}

VLC (cm3, \%): Volume and relative volume of the interstitial tissue occupied by Leydig cells, VCT (cm3, \%): Volume and relative volume of the interstitial tissue occupied by conjunctive tissue, VBV $(\mathrm{cm} 3, \%)$ : Volume and relative volume of the interstitial tissue occupied by blood vessels. Values with dissimilar letters within columns are significantly different ( $a$-b: $P<0.05$; a-c, c-e, e-g: $P<0.01$; a-e, a-g, c-g: $P<0.001)$. 
Table-5. Individual Leydig cell volume and their nucleus diameter according to the age of Sahraoui male camel

\begin{tabular}{|l|c|c|}
\hline Age (years) & $\mathbf{D}_{\mathbf{N y L C}}(\boldsymbol{\mu m})$ & $\mathbf{V}_{\mathbf{L C}}\left(\boldsymbol{\mu m}^{\mathbf{3}}\right)$ \\
\hline $\mathbf{2}(\mathbf{n}=\mathbf{6})$ & $2.05 \pm 0.37^{\mathrm{a}}$ & $1916.26 \pm 291.15^{\mathrm{a}}$ \\
\hline $\mathbf{3}(\mathbf{n}=\mathbf{6})$ & $2.15 \pm 0.35^{\mathrm{a}}$ & $1846.51 \pm 322.48^{\mathrm{a}}$ \\
\hline $\mathbf{4}(\mathbf{n}=\mathbf{6})$ & $6.50 \pm 1.52^{\mathrm{c}}$ & $6514.29 \pm 5926.99^{\mathrm{c}}$ \\
\hline $\mathbf{5}(\mathbf{n}=\mathbf{6})$ & $5.04 \pm 1.95^{\mathrm{c}}$ & $2842.70 \pm 2173.80^{\mathrm{c}}$ \\
\hline $\mathbf{7}(\mathbf{n}=\mathbf{6})$ & $5.68 \pm 0.46^{\mathrm{c}}$ & $4656.20 \pm 4550.84^{\mathrm{c}}$ \\
\hline $\mathbf{8}(\mathbf{n = 6})$ & $4.54 \pm 1.01^{\mathrm{c}}$ & $5383.22 \pm 4808.50^{\mathrm{c}}$ \\
\hline $\mathbf{1 0}(\mathbf{n = 6})$ & $6.03 \pm 1.09^{\mathrm{c}}$ & $4533.26 \pm 2035.38^{\mathrm{c}}$ \\
\hline$\geq \mathbf{1 3}(\mathbf{n}=\mathbf{6})$ & $5.32 \pm 1.09^{\mathrm{c}}$ & $5528.56 \pm 4081.43^{\mathrm{c}}$ \\
\hline
\end{tabular}

$D_{N y L C}(\mu m):$ Diameter of Leydig cell's nucleus, $V_{L C}$ $\left(\mu \mathrm{m}^{3}\right)$ : Individual Leydig cell volume. Values with dissimilar letters within columns are significantly different $(a-c: P<0.01)$.

Table-6. Seminiferous tubules histomorphometric parameters according to the age of Sahraoui male camel

\begin{tabular}{|l|c|c|c|c|}
\hline $\begin{array}{l}\text { Age } \\
\text { (years) }\end{array}$ & STD $(\boldsymbol{\mu m})$ & $\begin{array}{c}\text { DLTS } \\
(\boldsymbol{\mu m})\end{array}$ & STET $(\boldsymbol{\mu m})$ & $\begin{array}{c}\text { TLST } \\
(\mathbf{m})\end{array}$ \\
\hline $\mathbf{2}(\mathbf{n = 6})$ & $97.76 \pm$ & $38.16 \pm$ & $30.58 \pm$ & $1883.31 \pm$ \\
& $26.39^{\mathrm{a}}$ & $26.27^{\mathrm{a}}$ & $09.88^{\mathrm{a}}$ & $209.26^{\mathrm{a}}$ \\
\hline $\mathbf{3}(\mathbf{n = 6})$ & $106.44 \pm$ & $67.26 \pm$ & $27.16 \pm$ & $1290.81 \pm$ \\
& $50.15^{\mathrm{a}}$ & $44.01^{\mathrm{b}}$ & $14.58^{\mathrm{a}}$ & $33.83^{\mathrm{a}}$ \\
\hline \multirow{2}{*}{$\mathbf{4}(\mathbf{n = 6})$} & $139.59 \pm$ & $61.52 \pm$ & $44.42 \pm$ & $1278.76 \pm$ \\
& $34.35^{\mathrm{b}}$ & $28.37^{\mathrm{b}}$ & $13.81^{\mathrm{c}}$ & $136.36^{\mathrm{a}}$ \\
\hline \multirow{2}{*}{$\mathbf{5}(\mathbf{n = 6})$} & $152.52 \pm$ & $70.95 \pm$ & $42.61 \pm$ & $2505.89 \pm$ \\
& $48.66^{\mathrm{b}}$ & $30.22^{\mathrm{b}}$ & $14.48^{\mathrm{c}}$ & $135.68^{\mathrm{b}}$ \\
\hline \multirow{2}{*}{$\mathbf{7}(\mathbf{n = 6})$} & $169.88 \pm$ & $76.99 \pm$ & $48.21 \pm$ & $2738.97 \pm$ \\
& $38.51^{\mathrm{b}}$ & $43.86^{\mathrm{b}}$ & $10.85^{\mathrm{c}}$ & $69.32^{\mathrm{b}}$ \\
\hline \multirow{2}{*}{$\mathbf{8}(\mathbf{n = 6})$} & $155.87 \pm$ & $64.36 \pm$ & $40.63 \pm$ & $4875.36 \pm$ \\
& $94.13^{\mathrm{b}}$ & $51.27^{\mathrm{b}}$ & $14.81^{\mathrm{c}}$ & $30.38^{\mathrm{c}}$ \\
\hline \multirow{2}{*}{$\mathbf{1 0}(\mathbf{n = 6})$} & $181.32 \pm$ & $74.35 \pm$ & $52.38 \pm$ & $4421.23 \pm$ \\
& $42.90^{\mathrm{b}}$ & $38.87^{\mathrm{b}}$ & $12.61^{\mathrm{c}}$ & $89.5^{\mathrm{c}}$ \\
\hline \multirow{2}{*}{$\mathbf{1 3}(\mathbf{n = 6})$} & $126.29 \pm$ & $66.72 \pm$ & $31.45 \pm$ & $4826.46 \pm$ \\
& $74.28^{\mathrm{a}}$ & $53.50^{\mathrm{b}}$ & $14.29^{\mathrm{a}}$ & $56.66^{\mathrm{c}}$ \\
\hline
\end{tabular}

STD $(\mu m)$ : Seminiferous tubule diameter, DLST $(\mu m)$ : Seminiferous tubule lumen diameter, STET $(\mu \mathrm{m})$ : Seminiferous tubule epithelium thickness, TLST $(m)$ : Total length of seminiferous tubules. Values with dissimilar letters within columns are significantly different ( $a-b, b-c: P<0.05$; $a-c: P<0.01$ )

Animals aged 8 to 10 years' old display most active pre and post meiotic spermatogenesis. The first spermatids (Std) and spermatozoa (Sptz) are displayed in seminiferous tubules lumen from 4 years old class. The highest spermiogenesis level is observed in animals aged 7 and 8 years.

Therefore, the first significant increase of the Total Number of Germ cells (TGC) is enrolled at 4 years old $(68.00 \pm 75.74)$ and the maximum score is found in males at 10 years old $(333.60 \pm 161.80)$. The tubular fertility indexes display constant and progressive evolution towards 7 years old $(\mathrm{P}<0.01)$, where a high value of Germ/Srt and $\mathrm{ST}_{\mathrm{Std}: \mathrm{Sptz}}(\%)$ are observed which decrease significantly over to $9.64 \pm 2.32$ and $43 \%$ in $\geq 13$ years old category $(\mathrm{P}<0.05$, Supplementary figure 7).

Total number of Sertoli and Leydig cells per testes The first significant increase of the total numbers of Sertoli and Leydig cells is recorded at 4 and 5 years old, respectively ( $\mathrm{P}<0.01$; table 8$)$. They reach highest score between 8 and 10 years old $(\mathrm{P}<0.001)$, then they drop significantly in older animals $\geq 13$ years old $(\mathrm{P}<0.01, \mathrm{P}<0.001)$.

Table-7. Cell count of seminiferous tubules epithelium per transversal section and tubular fertility indexes according to Sahraoui male camel age

\begin{tabular}{|c|c|c|c|c|c|c|c|c|c|}
\hline $\begin{array}{c}\text { Age } \\
\text { (years) }\end{array}$ & sg & scyI & scyII & std & Sptz & srt & TGC & Germ/Srt & $\begin{array}{c}\mathbf{S T}_{\text {Std:Spt }} \\
{ }_{\mathbf{z}}(\%)\end{array}$ \\
\hline $2(n=6)$ & $30.80 \pm 9.04^{\mathrm{a}}$ & $9.37 \pm 4.48^{\mathrm{a}}$ & $0.00 \pm 0.00^{\mathrm{a}}$ & $0.00 \pm 0.00^{\mathrm{a}}$ & $0.00 \pm 0.00^{\mathrm{a}}$ & $16.27 \pm 5.70^{\mathrm{a}}$ & $40.17 \pm 12.58^{\mathrm{a}}$ & $2.53 \pm 0.46^{\mathrm{a}}$ & $00^{\mathrm{a}}$ \\
\hline $3(n=6)$ & $32.35 \pm 9.75^{\mathrm{a}}$ & $11.05 \pm 4.15^{\mathrm{a}}$ & $0.00 \pm 0.00^{\mathrm{a}}$ & $0.00 \pm 0.00^{\mathrm{a}}$ & $0.00 \pm 0.00^{\mathrm{a}}$ & $17.73 \pm 6.11^{\mathrm{a}}$ & $43.40 \pm 12.78^{a}$ & $2.55 \pm 0.58^{\mathrm{a}}$ & $12^{\mathrm{b}}$ \\
\hline $4(n=6)$ & $30.55 \pm 20.79^{\mathrm{a}}$ & $17.26 \pm 22.01^{\mathrm{a}}$ & $16.00 \pm 28.33^{b}$ & $3.39 \pm 7.95^{\mathrm{b}}$ & $0.79 \pm 2.52^{\mathrm{b}}$ & $12.87 \pm 5.35^{\mathrm{a}}$ & $68.00 \pm 75.74^{\mathrm{b}}$ & $5.05 \pm 4.82^{\mathrm{a}}$ & $68^{\mathrm{d}}$ \\
\hline $5(n=6)$ & $42.14 \pm 21.54^{b}$ & $30.94 \pm 23.13^{b}$ & $52.94 \pm 64.97^{c}$ & $5.69 \pm 9.67^{b}$ & $0.75 \pm 2.06^{\mathrm{b}}$ & $18.72 \pm 6.67^{\mathrm{a}}$ & $132.47 \pm 108.01^{\mathrm{b}}$ & $7.57 \pm 6.59^{\mathrm{a}}$ & $64^{\mathrm{d}}$ \\
\hline $7(n=6)$ & $53.00 \pm 21.98^{b}$ & $45.47 \pm 19.50^{\mathrm{b}}$ & $95.80 \pm 80.12^{\mathrm{c}}$ & $44.27 \pm 44.65^{\mathrm{d}}$ & $3.60 \pm 4.93^{\mathrm{c}}$ & $17.67 \pm 7.04^{\mathrm{a}}$ & $242.13 \pm 134.74^{\mathrm{b}}$ & $15.11 \pm 9.74^{\mathrm{c}}$ & $68^{\mathrm{d}}$ \\
\hline $8(n=6)$ & $74.74 \pm 32.59^{c}$ & $62.95 \pm 23.89^{c}$ & $90.79 \pm 58.96^{c}$ & $25.26 \pm 22.82^{\mathrm{c}}$ & $8.05 \pm 9.02^{\mathrm{d}}$ & $26.00 \pm 11.39^{b}$ & $261.79 \pm 119.10^{b}$ & $9.26 \pm 2.83^{\mathrm{b}}$ & $77^{\mathrm{d}}$ \\
\hline $10(n=6)$ & $98.40 \pm 47.10^{\mathrm{d}}$ & $79.80 \pm 48.20^{c}$ & $126.40 \pm 52.28^{\mathrm{d}}$ & $24.80 \pm 16.84^{c}$ & $4.20 \pm 6.26^{c}$ & $30.60 \pm 11.76^{b}$ & $333.60 \pm 161.80^{c}$ & $10.78 \pm 2.18^{b}$ & $73^{\mathrm{d}}$ \\
\hline$\geq 13(n=6)$ & $62.88 \pm 31.78^{\mathrm{b}, \mathrm{c}}$ & $56.13 \pm 21.32^{\mathrm{b}, \mathrm{c}}$ & $124.25 \pm 55.91^{\mathrm{d}}$ & $17.25 \pm 16.91^{b}$ & $4.63 \pm 7.58^{\mathrm{c}}$ & $26.88 \pm 7.30^{\mathrm{b}}$ & $265.13 \pm 107.43^{b}$ & $9.64 \pm 2.32^{\mathrm{b}}$ & $43^{c}$ \\
\hline
\end{tabular}

sg: spermatogonia, scyI: Spermatocysts I, scyII: Spermatocysts II, std: Spermatides, Sptz: Spermatozoa, srt: Sertoli cells, Tubular fertility indexes; TGC: Mean number of germ cells per seminiferous tubules section, Germ/Srt: Ratio of germ cells to Sertoli cells. Values with dissimilar letters within columns are significantly different (a$b, b-c, c-d: P<0.05 ; a-c, b-d: P<0.01 ; a-d: P<0.001)$ 
Table-8. Total number per testes of Sertoli cells and Leydig cells according to the age of Sahraoui male camel

\begin{tabular}{|l|c|c|c|}
\hline Age (years) & $\mathbf{N}_{\mathbf{L C} / \mathbf{T}}\left(\mathbf{. 1 0}^{\mathbf{9}}\right)$ & $\mathbf{S r t}_{\mathbf{T}} \mathbf{T}_{(\mathbf{C N})}\left(\mathbf{1 0}^{\mathbf{1 0}}\right)$ & $\begin{array}{c}\mathbf{S r t} / \mathbf{T}_{(\mathrm{TN})} \\
\left(\mathbf{1 0}^{\mathbf{1 0}}\right)\end{array}$ \\
\hline $\mathbf{2}(\mathbf{n}=\mathbf{6})$ & $1.18^{\mathrm{a}}$ & $19.36^{\mathrm{a}}$ & $5.70^{\mathrm{a}}$ \\
\hline $\mathbf{3}(\mathbf{n}=\mathbf{6})$ & $4.94^{\mathrm{a}}$ & $57.98^{\mathrm{c}}$ & $17.10^{\mathrm{a}}$ \\
\hline $\mathbf{4}(\mathbf{n}=\mathbf{6})$ & $5.26^{\mathrm{a}}$ & $89.16^{\mathrm{c}}$ & $26.22^{\mathrm{c}}$ \\
\hline $\mathbf{5}(\mathbf{n}=\mathbf{6})$ & $13.80^{\mathrm{c}}$ & $89.67^{\mathrm{c}}$ & $26.38^{\mathrm{c}}$ \\
\hline $\mathbf{7}(\mathbf{n}=\mathbf{6})$ & $10.47^{\mathrm{c}}$ & $101.88^{\mathrm{c}}$ & $30.0^{\mathrm{c}}$ \\
\hline $\mathbf{8}(\mathbf{n}=\mathbf{6})$ & $13.10^{\mathrm{c}}$ & $250.52^{\mathrm{f}}$ & $73.7^{\mathrm{f}}$ \\
\hline $\mathbf{1 0}(\mathbf{n}=\mathbf{6})$ & $27.00^{\mathrm{f}}$ & $310.17^{\mathrm{f}}$ & $91.23^{\mathrm{f}}$ \\
\hline$\geq \mathbf{1 3}(\mathbf{n}=\mathbf{6})$ & $12.79^{\mathrm{c}}$ & $211.46^{\mathrm{d}}$ & $62.20^{\mathrm{d}}$ \\
\hline
\end{tabular}

NLC /T .109: Number of Leydig cells per testes, Srt/T (CN).1010: Crude number of Sertoli cells, Srt/T(TN).1010: True number of Sertoli cells. Values with dissimilar letters within columns are significantly different ( $c-d: P<0.05$; a$c, d-f: P<0.01 ; a-d, a-f, c-f: P<0.001)$

\section{Discussion}

It is known that an accurate reproductive management of camel bull could reduce the level of herd infertility. The present study consists in assessment of the effect of camel bull age on its reproductive quality under extreme arid conditions; using a survey on male camel breeding practice and genital morpho-functional evaluation.

The average age at first mating (AFM) is lower than that observed by Mehari et al. (2013) in Ethiopian extensive camel herds. Half of the surveyed herders choose to allow their camel bulls to breed for the first time at 4 to 5 years old. These results corroborate the experimental findings conducted by Abd and Ibrahim (2014), Gherissi et al. (2018a; 2020) and Aubè et al. (2017) using different approaches to describe pubertyrelated changes (behavioral, morphological, hormonal and reproductive data). The rate of male camels which are allowed to breed at an age $>5$ years amount to $25 \%$. However, alarming value of $25 \%$ of camel bulls breed first time already at an age of 2-3 years. ElBahrawy et al. (2006) and El-Hassanein (2017) reported that full reproductive ability in the dromedary species was reached at 6-7 years old. Most authors implicate live weight, nutritional value of diet, calving conditions, month of birth, herd management (male ratio: female, weaning, welfare, herd mobility), neonatal complication and health status of camel calves as the main factors influencing age when they involved in herd reproduction (Al-Saiady et al., 2013, 2015). All young animals aged 2-3 years old are associated with higher number of mating for pregnancy per female and high mating time $(>20 \mathrm{~min}$ for $40 \%$ and $15-20 \mathrm{~min}$ for $60 \%$ ). However all animals aged $>$ 5years old show low mating time (standing over female stage $<15 \mathrm{~min}$ ). Longer time for mating in young animals compared to the adult and mature ones agrees with findings reported by Padalino et al. (2015) and Abdulmohsen et al. (2018). Furthermore, extending mating time in young dromedary bulls is often associated with a lack of libido or ejaculation failures (Al Qarawi et al., 2001).

The average culling age (CA) of male camel bulls is $15.30 \pm 2.47$ years; nevertheless $63.4 \%$ of camel herders cull breeding camels at age greater than 15 years old. Even a breeding camel bull can serve up to 20 years, it is not recommended to use it for mating more than 18 years old (Al Qarawi et al., 2001, Hafez and Hafez, 2001).

The ratio of male camel bull to breeding females (RMF) varied from 1:20 to 1:100 with an average of 1:40. This ratio increases in aged camel bull. Also, this parameter shows different score in relation to diverse breeding management practices at each country without leading particular relation of camel bull age and MFR ratio. The RMF varies from 1:10-30 females (Keskes et al., 2013) to 1:50-80 (Abdussamad et al., 2011). According to Al Qarawi (2005), herds with RMF $1:<25$ have an overall pregnancy rate of 95.29\%, whereas higher numbers of mating per day per bull reduce significantly sperm quality (Al Qarawi, 2005).

The morpho-functional exploration shows low PTV between 2 and 4 years old that increases and reaches a maximum value at 10 years old. Same observation is considered for the volume of the two testicular compartments (VIT and VST). These parameters have been significantly decreased in animals aged $\geq 13$ years old. Our results corroborate those recorded by $\mathrm{Al}$ Qarawi et al. (2000) among camels in Saudi Arabia with a 10-fold increase in testicular weight and volume between the age $<1$ year and 4.5 years. The testicular atrophy in older animals had been reported in some farm and companion animals (Barth, 2015). The spontaneous reduction in tubular relative volume (VST) in camels aged $\geq 13$ years is theoretically due to a reduction in the number, the length and the diameter of the tubules, or one or more combinations. Many of these aspects are investigated and reported later in this study.

Our results show a substantial increase with age of VIT compared to VST. In fact, VST/VIT ratio was significantly lower in prepubertal males $(\mathrm{P}<0.05)$ compared to pubertal and mature camels (ranged from 
$1.22 \pm 0.59$ to $1.64 \pm 0.40$ ) with some fluctuation as result of the effect of season, individual variations and histological processing technique. In addition, Gherissi et al. (2016) reported that interstitial tissue occupied a larger area and volume than those of seminiferous tubules especially in mature males with high reproductive activity, which validated findings of the current study.

The epididymis displays first significant morphological changes at 4 years old accompanied with significant increase of epididymal spermatozoa filling rate (ER). Epididymal pseudo-scarified columnar epithelium thickness (TEE) increases significantly from 7 years old. The most advanced epididymal morphology and the largest sperm reserve are observed in camel class aged 10 years old. Abd and Ibrahim (2014), reported that the sperm concentration level was significantly progressed with age in camels aged $<3$ years, 3 to 4 years and over 4 years. The highest morphologically abnormal sperm was observed in $<3$ years old category (Abd and Ibrahim, 2014).

The Leydig cells occupy predominant volume of the testicular endocrine compartment. It varies considerably in relation to individual cell volume, total number and relative volume of Leydig cells $\left(\mathrm{V}_{\mathrm{LC}}, \mathrm{N}_{\mathrm{LC}}\right.$ $/ \mathrm{T}$ and VLC \%). Our results also show that camels aged 2 to 3 years display low $\mathrm{V}_{\mathrm{LC}}$ and $\mathrm{D}_{\mathrm{NyLC}}$ that increase significantly towards 4 years old. These two parameters rise just before the significant increase of VIT occurring at 5 years old. This pattern of change is similar to developmental features reported previously by Al Qarawi et al. (2001) who suggested a significant increase of Leydig cell size at 3 years old, accompanied at this age by increased proportion of relative volume of interstitial tissue compared to that of seminiferous tubules. The recorded VLC (\%) at puberty agrees with that reported by Ibrahim et al. (2016) in Egyptian camels. The average number of Leydig cells at this stage varies from $5.26 \times 10^{9}$ to 13.8 $\mathrm{x} 10^{9}(\mathrm{P}<0.01)$. This is in agreement with the fact that common hypertrophy and/or hyperplasia of the interstitial cells with increased volume of the intertubular tissue are secondary to elevation in gonadotropins at puberty (Suttie et al., 2017). Hence, the morphological characteristics of Leydig cells and interstitial blood vessels volume during the puberty and post-puberty support the statement of their parallel activity with spermatogenesis (exocrine compartment), epididymal spermatozoa content level and increased testicular volume.

Ones the strong effect of age on testicular morphometry was considered, our results revealed precisely a significant changes of exocrine parenchyma organization showing evidences regarding tubular density, germinal epithelium proliferation and seminiferous tubules morphometry. The mean diameters of the seminiferous tubules in adult camels are close to those reported previously by Ibrahim et al. (2016) and Gherissi et al. (2016). Male camels aged 4 years old exhibit first significant expansion of STD and STET. While the significant increase of DLST and TLST is recorded at 3 and 5 years old, respectively. Similarly, Al Qarawi et al. (2001) reported significant increase of seminiferous tubules measurements around 4.5 years old $(\mathrm{P}<0.01)$ that reached maximum values at 7 year old males.

The first significant raise in $\mathrm{Srt} / \mathrm{T}_{(\mathrm{TN})}$ is recorded at 4 years old and reaches peak score towards 8 years before falling significantly in animals ages $\geq 13$ years. The Sertoli cells number development over postnatal through to maturity confirms pattern of change reported previously in one humped camel species ( $\mathrm{Al}$ Qarawi et al., 2001). This author mentioned that average number of Sertoli cells per 100 sections increases significantly in males aged 5.5 years before reaching the pick at the age of 7.5 years (Al Qarawi et al., 2001). The morphometric description of the Sertoli cells at the onset of puberty coincides with the increase of Sertoli cell size, development of extensive Sertoli cell cytoplasmic processes and establishment of Sertoli cells blood-testis barrier (Mruk and Cheng, 2015). We admit that ultimate increase of Sertoli cells number at puberty creates a specialized microenvironment for meiotic spermatocytes and post-meiotic spermatids and thereafter throughout adulthood. Particularly at puberty, these cells ensure the metabolism of glucose to pyrovate and lactate as the primary source of energy to germ cells (Chojnacka et al., 2016).

The seminiferous tubules (ST) sections with only younger generation cells (spermatogonia and spermatocytes I) are recorded in animals aged 2 to 3 years old. Yet, spermatocytes II, spermatids and spermatozoa within ST sections were evident from 4 years old. We speculate that Sertoli cell differentiation can orchestrate complete mandatory spermatogenesis form this age leading to histological pubertal features. Similar descriptions are given by Koskenniemi et al. (2017) during the early puberty stage and by $\mathrm{Al}$ Qarawi et al. (2001) in male camels form Saudi Arabia. Testes form animals aged 3 years old show first differentiation of the undifferentiated support 
cells into Sertoli cells and the secondary spermatocytes into spermatids with appearance of spermatozoa in seminiferous tubules (Al Qarawi et al. 2001). The high spermatogonial proliferation and expansion of meiotic and haploid germ cells in mature males (7 to 8 years old) implies to largest and fastest growth of the testes and lead to significant increase in the diameter and volume of the seminiferous tubules.

The present study shows that testicular spontaneous degeneration occurs in older camel bulls ( $\geq 13$ years). It reaches Sertoli Cell-Only Syndrome level in some cases (SCOS; Supplementary figure 7). The testicular atrophy and degeneration reduce the tubular and interstitial tissue density (VST, VIT \%) leading to increase peri and inter tubular empty spaces and fibrosis with significant decrease of $\mathrm{N}_{\mathrm{LC} / \mathrm{T}}$. The ER and the TEE are decreased at this stage of reproductive life. In addition; STD, STET, Srt/T $\mathrm{T}_{(\mathrm{TN})}$ and tubular fertility indexes have been dropped significantly. These results are in line with different degrees of irreversible hypospermatogenesis reported in aged mammalian species (Campos-Silva et al., 2015; Bhanmeechao et al., 2018). Also, degenerative reactions on the testes of aged camels appear to be amplified by extensive breeding conditions as suggested particularly in rams and bulls (Fernandes et al., 2010).

The results of this study provide good evidence that the establishment of spermatogenesis, its maintenance and its fluctuation in different pubertal stages of male dromedaries are associated with pronounced changes in the morphology and functional structure of the testes. Several authors indicated that these variations are in relation with the body weight growth and body condition score (DWG and BCS), themselves in close relationship with the consumption index (CI), the nutritional status of the rations, climatic conditions and geographical location, birth season of camel calves and the livestock management system (Mohamed, 2007). Indeed, these interdependent factors are determinant for pubertal age, fecundity of the camel bull, age to sexual maturity and culling age. The available data lead to better understanding postnatal reproductive development of the male camels and eventually the infertility constraints depending on them (age to choice camel bull, ratio male/females, culling age). The histomorphometry analysis shows that the puberty in male camels is undertaking between 4 and 5 years old. At this stage, we highlighted high proliferation of germ and Leydig cells with achievement of ultimate spermatogenesis stages and high filling rate of epididymis. These findings corroborate the substantial proportion of camel herders who engage their camel bulls for breeding between 4 and 5 years old. However, there are often some difficulties in this age group leading to mainly higher number of mating and a longer mating time. These reproductive traits are greatly improved in animals aged $>6$ years. This category shows the highest level of camel testicular activity displaying the maturity stage towards 8 years old. Finally, camel bull reform at 13 years old as threshold is to be recommended before the physiological reproductive potential drops in relation to high level of genital involution and low tubular fertility indexes observed from this age.

\section{Conclusion}

Compared to relatively long life expectancy of the camel species (40 to 50 years), male camel show delayed puberty (4 to 5 years), short duration of maximum activity corresponding to sexual maturity (5 years) and the early culling age (13 years) suggesting serious management difficulties and contribute to low reproductive performance of camel herds. In order to pursuit a efficient breeding age of camel bull, it is recommended to improve livestock conditions of the potential future breeder animals, train camel herders for efficient monitoring of reproductive processes and use of reproductive biotechnologies for wide and fast spread of camel bull genetic potential.

\section{Disclaimer: None. \\ Conflict of Interest: None. Source of Funding: None.}

\section{References}

Abd AA and Ibrahim NS, 2014. Effect of age and season on the Epididymal Sperm and testosterone level in camel (Camelus dromedarius). Iraqi. J. Vet. Sci. 38(1): 24 -29.

Abdulmohsen M, Al-Shami SA, Al-Sultan S and AlEkna M, 2018. Evaluation of mating and the causes of noises at night in small dromedary camel herds. Emir. J. Food. Agric. 30(8): 709-714.

Abdussamad AM, Holtz W, Gauly M, Suleiman MS and Bello MB, 2011. Reproduction and breeding in dromedary camels: insights from pastoralists in some selected villages of the Nigeria-Niger corridor. Livestock Res. Rural. Dev. 23: Article No. 178. 
Al Qarawi AA, Abdel-Rahman HA, El-Belely MS and El-Mougy SA, 2000. Age-related changes in plasma testosterone concentrations and genital organs content of bulk and trace elements in the male dromedary camel. Anim. Reprod. Sci. 62: 297-307.

Al Qarawi AA, Abdel-Rahman HA, El-Belely MS and El-Mougy SA, 2001. Intratesticular morphometric, cellular and endocrine changes around the pubertal period in dromedary camels. Vet. J. 162: 241-249.

Al Qarawi AA, 2005. Infertility in the dromedary bull: a review of causes, relations and implications. Anim. Reprod. Sci. 87: 73-92.

Al-Saiady MY, Mogawer HH, Al-Mutairi SE, Bengoumi M, Musaad A, Gar- Elnaby A and Faye B, 2013. Effect of different feeding system on body weight, testicular size developments, and testosterone level in prepubertal male camel (Camelus dromedarius). Afr. J. Agric. Res. 8(22): 2631-2636.

Al-Saiady MY, Mogawer HH, Al-Mutairi ES, Bengoumi M, Musaad A, Gar-ElnabyA and Faye B, 2015. Factors affecting feed intake, body weight, testicular size, and testosterone, follicle stimulating hormone (FSH) and luteinizing hormone (LH) serum concentrations in peripubertal male camels. Afr. J. Agric. Res. 10(14): 1709-1713.

Aubè L, Fatnassi M, Monaco D, Khorchani T, Lacalandra GM, Hammadi $\mathrm{M}$ and Padalino B, 2017. Daily rhythms of behavioral and hormonal patterns in male dromedary camels housed in boxes. PeerJ. 5: e3074.

Barth A, 2015. Testicular degeneration. In: Hopper RM, ed. Bovine Reproduction. Ames, IA: Wiley, pp. 103-108.

Bhanmeechao C, Srisuwatanasagul S and Ponglowhapan S, 2018. Age-related changes in interstitial fibrosis and germ cell degeneration of the canine testis. Reprod. Dom. Anim. 53(Suppl. 3): 37-43.

Campos-Silva P, Furriel A, Costa WS, Sampaio FJ and Gregório BM, 2015. Metabolic and testicular effects of the long-term administration of different high-fat diets in adult rats. Int. Braz. J. Urol. 41(3): 569-575.

Chojnacka K, Zarzycka M, Hejmej A, Mruk DD, Gorowska E, Kotula-Balak M, Klimek M and Bilinska B, 2016. Hydroxyflutamide affects connexin 43 via the activation of PI3K/Aktdependent pathway but has no effect on the crosstalk between PI3K/Akt and ERK1/2 pathways at the Raf-1 kinase level in primary rat Sertoli cells. Toxicol. In Vitro. 31: 146-157.

Dhakal P, Hirama A, Nambo Y, Harada T, Sato F, Nagaoka K, Watanabe G and Taya K, 2012. Circulating pituitary and gonadal hormones in spring-born Thoroughbred fillies and colts from birth to puberty. J. Reprod. Dev. 58: 522-530.

El-Bahrawy KA, El-Hassanien EE, Fateh El-bab AZ and Zeitoun MM, 2006. Semen Characteristics of the Male Camel and its Freezability after Dilution in Different Extenders. International Scientific Camel Conference, 9-11 May, El-Qaseem, KSA. pp. 2037-2053.

El-Harairy MA and Attia KA, 2010. Effect of age, pubertal stage and season on testosterone concentration in male dromedary camel. Saudi. J. Biol. Sci. 17: 227-230.

El-Hassanein ES, 2017. Prospects of improving semen collection and preservation from elite Dromedary camel breeds. World Vet. J. 7(2): 47-64.

FAOSTAT, 2018. Accessed from: http://faostat.fao.org/

Faye B, 2016. The camel, new challenges for a sustainable development. Trop. Anim. Health. Prod. 48: 689-692.

Fernandes JMO, Ruangsri J and Kiron V, 2010. Atlantic cod piscidin and its diversification through positive selection. PLoS ONE. 5. e9501.

Gherissi DE, 2015. Survey on camel husbandry in OuedRigh region, South East of Algeria: production systems and reproductive performances. Poster session presented at: 4th ISOCARD Conference in 8-12 June 2015 at Kazakhstan.

Gherissi DE, Afri-Bouzebda F, Bouzebda $\mathrm{Z}$ and Lamraoui R, 2016. Testicular morphology and stereological evaluation of the seminiferous tubules around the rutting season of Sahraoui Dromedary camel. Glob. Vet. 17(6): 568-576.

Gherissi DE, Afri-Bouzebda F, Bouzebda Z and Lamraoui R, 2014. Morphometric variations and endocrine changes of the one-humped male camel in relation to reproductive activity. Rumin. Sci. 3(1): 9-18.

Gherissi DE, Afri-Bouzebda F, Bouzebda Z, 2018b. Seasonal changes in the testicular morphology and interstitial tissue histomorphometry of Sahraoui camel under Algerian extreme arid conditions. Biol. Rhythm. Res. 49(2): 1744-4179. 


\section{Gherissi Djalel Eddine et al.}

Gherissi DE, Afri-Bouzedba F, Bouzebda $\mathrm{Z}$ and Bonnet X, 2018a. Are female camels capital breeders? Influence of seasons, age, and body condition on reproduction in an extremely arid region. Mamm. Biol. 93: 124-134.

Gherissi DE, Monaco D, Bouzebda Z, AfriBouzebda F, Gaouar SSB and Ciani E, 2020. Camel herds' reproductive performance in Algeria: objectives and thresholds in extreme arid conditions. J. Saudi Soc. Agric. Sci. In press.

Hafez ESE and Hafez B, 2001. Reproductive parameters of male Dromedary and Bactrian camels. Arch. Androl. 46: 85-98.

Ibrahim MA, Abd-El-Rahman HMA, Rawash ZM and El-Metwally AE, 2016. Studies on some biochemical, hormonal, histopathological and seminal characters in relation to rutting and nonrutting season in camels. Alex. J. Vet. Sci. 49(2): 189-202. doi: 10.5455/ajvs.226663.

Keskes S, Ibrahim M, Tessema TS, Tamir B, Regassa F, Kassa T and Dawo F, 2013. Production systems and reproductive performances of Camelus dromedarius in Somali regional state, eastern Ethiopia. J. Agric. Environ. Int. Develop. 107: 243266.

Koskenniemi JJ, Virtanen HE and Toppari J, 2017. Testicular growth and development in puberty. Curr. Opin. Endocrinol. Diabetes. Obes. 24: 215224.

Mehari Y, Gebru G and Mekuriyaw Z, 2013. Reproductive performance of camels in Babilie and Kebribeyah districts of the Jijiga zone, Somali Region, Ethiopia. International Scientific Conference of Camel Research and Production
(ISCCRP), Khartoum-Sudan, 17th-18th April 2013. p. 59.

Mirkena T, Walelign E, Tewolde N, Gari G, Abebe G and Newman S, 2018. Camel production systems in Ethiopia: a review of literature with notes on MERS-CoV risk factors. Pastoralism. 8: 30.

Mohamed MI, 2007. Evaluation of Growth Performance for Growing Maghraby Camel Fed on Unconventional Feed. Int. J. Agric. Biol. 9(1): 1821.

Mruk DD and Cheng CY, 2015. The mammalian blood-testis barrier: Its biology and regulation. Endocr. Rev. 36: 564-591.

Padalino B, Monaco D and Lacalandra GM, 2015. Male camel behavior and breeding management strategies: how to handle a camel bull during the breeding season? Emir. J. Food. Agric. 27: 338349.

Suttie AW, Leininger JR and Bradley AE, 2017: Boorman's Pathology of the Rat: Reference and Atlas. Academic Press; 2 edition, pp. 564-571.

Tibary A and Anouassi A, 1997. Theriogenology in Camelidae. Abu Dhabi printing and publishing Company, Mina Abu-Dhabi, UAE.

\section{Contribution of Authors}

Gherissi DE: Designed the study, performed experiments, data analysis, manuscript writing Boukhili M: Contributed to the experiments Gherissi A: Analysis of the data, manuscript writing. 